\title{
PSICOEDUCAÇÃO DAS NECESSIDADES BÁSICAS EMOCIONAIS AOS PAIS/ CUIDADORES E RELAÇÃO COM HABILIDADES SOCIAIS
}

\author{
PSICOEDUCACIÓN DESDE LAS NECESIDADES EMOCIONALES BÁSCIAS A LOS \\ PADRES/ CUIDADORES Y RELACIÓN COM LAS HABILIDADES SOCIALES
}

\author{
PSYCHOEDUCATION FROM BASIC EMOTIONAL NEEDS TO PARENTS / \\ CAREGIVES AND RELATION WITH SOCIAL SKILLS
}

\author{
Tatiani Justin Witt CARDOSO ${ }^{1}$ \\ Rafaela Fava de QUEVEDO ${ }^{2}$
}

RESUMO: Trabalhar habilidades sociais em crianças e adolescentes pode diminuir ou evitar problemas de comportamento e transtornos psicológicos. Contudo, propõe-se que um trabalho com cuidadores com psicoeducação com um conhecimento desenvolvido pela terapia do esquema sobre necessidades básicas emocionais pode contribuir. Esta revisão de literatura se justifica pela escassez de pesquisas sobre essa temática. Para tanto, utilizou-se estudos publicados na base de dados da Scielo e Google Acadêmico, integrando 30 estudos. Os resultados apontaram que o desenvolvimento emocional saudável e as habilidades sociais têm relação com o convívio com pais ou cuidadores, os quais podem ou não possuir habilidades sociais parentais desenvolvidas. Contudo, programas com psicoeducação a pais resultam em alteração positiva nas habilidades educativas parentais relacionadas à alteração positiva nas habilidades sociais dos filhos. Este estudo forneceu subsídios para refletirmos sobre importância da psicoeducação neste contexto.

PALAVRAS-CHAVE: Eficácia da psicoeducação. Habilidades sociais. Estilos parentais.

RESUMEN: Trabajar con habilidades sociales en niños y adolescentes puede disminuir o evitar problemas de conducta y trastornos psicológicos. Sin embargo, se propone que puede contribuir un trabajo con cuidadores con psicoeducación con un conocimiento desarrollado por la terapia de esquemas sobre necesidades emocionales básicas. Esta revisión de la literatura se justifica por la escasez de investigaciones sobre este tema. Para ello se utilizaron estudios publicados en la base de datos de Scielo y Google Scholar, integrando 30 estudios. Los resultados mostraron que el desarrollo emocional saludable y las habilidades sociales están relacionadas con la convivencia con los padres o cuidadores, quienes pueden o no haber desarrollado habilidades sociales de los padres. Sin embargo, los programas con psicoeducación para padres dan como resultado un cambio positivo en las habilidades educativas de los padres relacionado con un cambio positivo en las habilidades sociales de los niños. Este estudio proporcionó subsidios para reflexionar sobre la importancia de la psicoeducación en este contexto.

\footnotetext{
${ }^{1}$ Capacitar Centro de Ensino Superior (CAPACITARNH), Osório - RS - Brasil. Especialista em Terapias Cognitivas Comportamentais. ORCID: https://orcid.org/0000-0002-4887-5987. E-mail: E-mail: tatiani.justin@gamil.com

${ }^{2}$ Universidade do Vale do Rio dos Sinos (UNISINOS), São Leopoldo - RS - Brasil. Mestrado em Psicologia clínica. ORCID: https://orcid.org/0000-0001-8968-8064. E-mail: rafaelafaq@msn.com
} 
PALABRAS CLAVE: Efectividad de la psicoeducación. Habilidades sociales. Estilos de crianza.

ABSTRACT: Working with social skills in children and adolescents can decrease or avoid behavior problems and psychological disorders. However, it is proposed that a work with caregivers with psychoeducation with a knowledge developed by the schema therapy on basic emotional needs can contribute. This literature review is justified by the scarcity of research on this topic. For this purpose, studies published in the database of Scielo and Google Scholar were used, integrating 30 studies. The results showed that healthy emotional development and social skills are related to living with parents or caregivers, who may or may not have developed parental social skills. However, programs with psychoeducation for parents result in a positive change in parental educational skills related to a positive change in children's social skills. This study provided subsidies to reflect on the importance of psychoeducation in this context.

KEYWORDS: Effectiveness of psychoeducation. Social skills. Parenting styles.

\section{Introdução}

A comunicação e a socialização, geralmente, iniciam com pais e cuidadores. As “estratégias de socialização utilizadas pelos pais são denominadas por alguns autores de práticas educativas parentais" (DEL PRETE; DEL PRETE, 2003, p. 21). Essas práticas podem desenvolver nas crianças e adolescentes comportamentos pró-sociais ou antissociais. Vai depender da frequência e da intensidade em que são adotadas determinadas estratégias educativas e de "fatores biológicos, evolutivos e de aprendizagem" (DEL PRETE; DEL PRETE, 2003, p. 22). Aspectos como comunicação verbal e não verbal, empática, comportamento assertivo, passivo e agressivo são abordados por Portella (2011) e pontuados como consequências ao tempo e à forma das conquistas dos objetivos e das necessidades, ligadas às habilidades sociais. $\mathrm{O}$ autor ainda cita o feedback em relação ao comportamento como uma possibilidade de ajuste às demandas de interação quando necessário, trazendo como exemplos elogios e críticas (PORTELA, 2011).

Vários autores tentam definir habilidades sociais, o que faz com que surjam várias definições como o conteúdo (o que faz), as consequências (reações provocadas nos outros) e ambos. Em geral, “espera-se que o comportamento social hábil gere mais reforço positivo que negativo" (CABALLO, 2003, p. 05). Desmembrando o conteúdo sobre habilidades sociais, o autor se depara com o conceito de competência, ou seja, o quão adequada é a atuação de uma pessoa para a realização de uma tarefa. Por habilidades, compreende como capacidades específicas para que se execute, competentemente, uma determinada tarefa, podendo ser 
inatas ou adquiridas. Assim como compreende por social o interesse na conduta (CABALLO, 2003). A conduta social é entendida como os outros tratam o indivíduo, sendo o reflexo do seu comportamento em relação a eles. Ainda relevante, o prejuízo nas habilidades sociais pode causar dificuldades a nível social, laboral e da saúde, o que pode colaborar para o desenvolvimento de transtornos psicológicos ou psiquiátricos (KNAPP et al., 2007).

Pesquisas vêm sendo realizadas no sentido de investigar a relação entre repertório parental de habilidades sociais e o desenvolvimento de habilidades sociais em crianças e adolescentes. Considerações foram feitas relacionando habilidades sociais desenvolvidas com menor quantidade de problemas de comportamento, com pais que expressam sentimentos, enfrentam e são mais habilidosos na comunicação. Uma intervenção em grupo cujos alguns assuntos discutidos foram expressão de sentimentos, interação comunicativa, negociação, estabelecimento de regras, verificou aumento na assertividade em práticas parentais e diminuição nos comportamentos problema (BOLSONI-SILVA; SILVEIRA; MATURANO, 2008).

Ao considerar estudos como os citados, Young (2008, p. 21) "desenvolveu a terapia do esquema como uma abordagem sistemática que amplia a Terapia cognitivo-comportamental, integrando técnicas de várias escolas diferentes de terapia". Dando maior ênfase do que a Terapia Cognitivo-Comportamental (TCC) ao desenvolvimento infantil e adolescente, assim como para os estilos parentais e emoções. Na terapia do esquema todo ser humano tem esquemas e maneiras de enfrentamento a esses esquemas. Sendo o esquema resultado das experiências no desenvolvimento infantil, sendo reforçadas ao longo do desenvolvimento. Parte desse estudo culminou na importância da satisfação das necessidades básicas emocionais, sendo elas: os vínculos seguros; a autonomia e competência; os limites realistas; as necessidades e suas emoções validadas; os sentimentos expressos de forma assertiva e a espontaneidade das escolhas. Para ele o ser humano em desenvolvimento passa por um processo de maturação, em que embora algumas pessoas tenham mais necessidades do que outras por diferenças biológicas inatas (temperamento), as necessidades básicas emocionais são cruciais para o desenvolvimento emocional saudável. Assim, é possível desenvolver modos mais assertivos consigo e com quem convive se supridas as necessidades suficientemente, tendo o foco adequado nas suas necessidades e as dos outros, sendo assertiva e espontânea nos seus sentimentos e escolhas.

Este artigo se justifica pela necessidade da realização de um estudo mais aprofundado acerca da psicoeducação das necessidades básicas emocionais a pais ou cuidadores e sua identificação por estes, a fim de poder valer-se deste conhecimento para beneficiar o seu 
desempenho, podendo resultar em desenvolvimento de habilidades sociais nos filhos. Dentro da abordagem cognitivo-comportamental, a psicoeducação promove conhecimentos psicológicos relevantes ao indivíduo. O conhecimento tende a desenvolver uma sensação de controle da situação, à medida que passam a ter conhecimento passam a observar como funcionam podendo assim melhorar a motivação para a mudança (KNAPP et al., 2007).

Um estudo recente sobre psicoeducação retrata trabalhos realizados em diversas modalidades em que revelam uma melhora na autonomia, lazer e relacionamentos interpessoais (NOGUEIRA; CRISOSTOMO; SOUZA; PRADO, 2017). O que também pode ser aplicada a cuidadores (LEMES; NETO, 2017). Sendo assim, o objetivo geral deste estudo, foi investigar a respeito da psicoeducação das necessidades básicas emocionais da abordagem cognitivo-comportamental focada nos esquemas com intuito de aplicá-la como mais uma ferramenta de auxílio ao desenvolvimento de habilidades sociais de criança ou adolescentes.

\section{Método}

A investigação teve caráter bibliográfico, buscando informações em obras de autores que se dedicaram a este assunto bem como consulta em estudos científicos publicados na base de dados da Scielo e Google Acadêmico. Utilizou- se das seguintes expressões para a busca dos artigos: "eficácia da psicoeducação" e "habilidades sociais e estilos parentais". O acesso ocorreu entre maio a agosto de 2019. Foram encontrados na base de dados Scielo oito estudos relacionado à expressão "eficácia da psicoeducação" (sendo excluídos quatro, restando quatro) e 12 relacionados a "habilidades sociais e estilos parentais" (sendo excluídos cinco, restando sete). Na base de dados Google Acadêmico foram buscados os primeiros 10 mais relevantes e os primeiros 10 mais atuais de ambas as expressões sendo excluídos da expressão "eficácia da psicoeducação" 12 estudos, restando oito e, "habilidades sociais e estilos parentais" excluídos nove estudos, restando 11. Resultando em 12 estudos sobre a "eficácia da psicoeducação" e 18 estudos sobre "habilidades sociais e estilos parentais". Sendo integrados a este trabalho 30 estudos que melhor respondiam os questionamentos levantados. Classificando em duas categorias: Eficácia da psicoeducação e relação entre habilidades sociais e estilos parentais. 


\section{Resultados e Discussão}

\section{Eficácia da psicoeducação}

Na revisão integrativa de literatura de Silva, Sá e Souza (2018) sobre programas de psicoeducação a cuidadores encontrou-se resultados positivos na sobrecarga e no bem-estar. Aponta para melhora na qualidade de vida, na comunicação, na colaboração, na forma de lidar com os problemas e atividades diárias e, diminuindo a desesperança ao entender o comprometimento da demência. Da mesma forma, Cuevas-Cancino e Moreno-Pérez (2017), consideram que a psicoeducação em intervenção em grupo pode proporcionar redes de apoio, abre mais formas de enxergar as situações e acrescenta aquisição de habilidades. Brandão e Matos (2015) realizaram uma revisão de literatura sobre a eficácia das intervenções psicoeducativas e cognitivas comportamentais em grupo a mulheres com cancro de mama. Os resultados dos estudos revelam melhoria na qualidade de vida, redução de ansiedade e depressão, maior percepção de apoio, dentre outros.

Um ensaio clínico randomizado de Valente, Moreira, Ferigolo e Barros (2019) para mudar as práticas de uso de drogas dos pais. Observou maior eficácia em intervenção breve do que em psicoeducação para as mudanças positivas nas práticas dos pais em monitoramento, abuso físico, punição não condizente. Lemes e Neto (2017) a partir de uma revisão sistemática de literatura sobre aplicações da psicoeducação em saúde constataram a sua aplicabilidade em diferentes tipos de doenças. Com a psicoeducação ligada ao suporte e apoio ao cuidador. Um estudo de caso com um grupo de doze portadores de TAB com psicoeducação, Menezes e Souza (2012) apresentou um papel fundamental na compreensão para a aceitação, adesão ao tratamento e melhora na qualidade das atividades diárias. Através de uma revisão sistemática de 27 artigos sobre a intervenção com psicoeducação a cuidadores de idosos com demência, Lopes e Cachioni (2012), encontrou contribuição significativa na melhoria do bem-estar, aquisição de estratégias de enfrentamento, senso de autoeficácia, diminuindo sentimentos e pensamentos disfuncionais.

Coelho (2012) em seu estudo sobre contributos da psicoeducação sobre a competência emocional em professores apresenta resultados positivos em relação à saúde mental, autoconsciência e automotivação, assim como a empatia e gestão de emoções. Pellegrini (2010) em sua dissertação através de um estudo randomizado controlado por um ano sobre o impacto da psicoeducação na recuperação sintomática e funcional dos pacientes bipolares. 
Verificou que a psicoeducação pode impactar positivamente no bem-estar por promoção de hábitos saudáveis, baixa adesão ligada a baixo apoio familiar.

\section{Práticas parentais e sua relação com o desenvolvimento de habilidades sociais em crianças e adolescentes}

Salvo, Silvares e Toni (2005) realizaram um estudo sobre práticas educativas como predição de problemas de comportamento, assim como competência social. Os autores constataram relação entre problemas de comportamento e competência social, ligados à negligência e a monitoria inadequada (falta ou excesso). Coelho e Murta (2007) descreveram uma experiência de treinamento de pais em grupo com cinco mães e dois pais de 20 seções de 90 minutos semanais com técnicas comportamentais. A avaliação indicou resultados positivos nas habilidades sociais educativas parentais, assim como mudanças positivas nos filhos. Vilas-Boas e Bolsoni-Silva (2010) em sua pesquisa com 43 mães sobre habilidades sociais educativas e a relação com comportamento de pré-escolares identificaram a relação entre as habilidades das mães e habilidades das crianças, assim como práticas negativas com problemas de comportamento.

Um estudo de habilidades educativas parentais relacionando a comportamentos préescolares com 20 mães com filhos com problemas de comportamento e 20 sem problemas de comportamento de autoria de Leme e Bolsoni-Silva (2010) revelou um indicativo positivo entre práticas educativas parentais com expressão de sentimentos, enfrentamento assim como comunicação e poucas práticas negativas a filhos mais habilidosos e com menos problemas de comportamento. Bolsoni-Silva e Loureiro (2019) realizam um estudo de caso controle com 18 mães de filhos com problemas de comportamento e 18 sem. As correlações associaram práticas positivas a comportamentos habilidosos e as práticas negativas aos problemas de comportamento.

Martins et al. (2014) em seu estudo para verificar a relação entre as práticas parentais, o desempenho escolar e as habilidades sociais com a participação de 117 pais indicaram que as práticas parentais com afeto e controle adequados têm relação positiva com a competência acadêmica e o controle psicológico uma relação negativa com habilidades sociais. Mondin (2008) em seu estudo sobre práticas parentais e efeitos nos filhos descreveu quatro estilos parentais que culminavam em resultados diferentes nos filhos. Logo, pais autoritativos em que predomina exigência e responsividade adequada, com comunicação clara e aberta e com respeito mútuo possibilita a desenvolver habilidades sociais nos filhos. O estilo autoritário característico de autocontrole e baixa responsividade prezam respeito e obediência, utilizando 
com mais frequência punição, não valorizando o diálogo, a autonomia e as opiniões dos filhos. $\mathrm{O}$ indulgente com baixo controle e alta responsividade, sem estabelecimento de regras e limites. O negligente com baixo controle e responsividade, não se envolvem com os filhos, nem exigem e nem são afetivos. Técnicas coercivas utilizadas por alguns estilos parentais com mais frequência como autoritário e falta de afeto, como também no negligente, podem gerar nas crianças medo, raiva e ansiedade e dificuldade de compreender a situação e o motivo da mudança no comportamento.

Bolsoni-Silva (2016) em seu estudo sobre associação entre habilidades sociais, práticas parentais e recursos do ambiente e depressão materna, ligados a problemas internalizantes com 32 mães com filhos com problemas e 32 sem, pareadas por sexo e escolaridade. Havendo mais frequência de práticas negativas no grupo com problemas e habilidades sociais no outro grupo. Mariano e Bolsoni-Silva (2016) realizaram um estudo comparando as práticas educativas dos professores com habilidades sociais e problemas de comportamento em 283 crianças. Professores que apresentaram dificuldade em lidar com os problemas parecem reforçar os problemas, promovendo a manutenção dos problemas e ainda podendo intensificá-los, enquanto professores com comportamentos habilidosos parecem promover habilidades nas crianças por imitação e emissão de feedback. Del Prette e Del Prette (2018) em seu estudo sobre a relação entre habilidades sociais e a análise do comportamento apresenta um campo teórico prático e pesquisas produzidas a partir de 1970, que descrevem a forma como ocorre a aprendizagem dos comportamentos desejáveis e indesejáveis pode ser por "três processos básicos (e suas combinações), demonstrados em experimentos controlados: instrução (controle por regras), consequenciação e imitação/modelação [...] podem ser simultâneos ou isolados, em sequência planejada ou aleatória" (DEL PRETE; DEL PRETE, 2018. p. 45).

Oliveira e Loureiro (2019) em uma validação de entrevista de práticas educativas para professores verificaram um "conjunto de habilidades dos professores que poderão melhorar as habilidades sociais infantis e reduzir problemas de comportamento, tais como a competência em conversar... estabelecer regras/limites" (OLIVEIRA; LOUREIRO, 2019, p. 9), assim como validar os bons comportamentos e ser afetuoso, dentre outros. Considerando a escola como um ambiente de possível identificação precoce e prevenção.

Uma revisão sistemática de literatura de Guisso, Bolze e Viera (2019), sobre práticas e programas de treinamento parental indicaram mudanças de comportamento parental e melhora nos comportamentos dos filhos. Reduzindo práticas negativas e aumentando as positivas. Sendo aplicado em diversas realidades como creches, escolas, hospitais, sites, 
dentre outros. $\mathrm{O}$ autor coloca que a análise dos estudos aponta "para avaliações positivas dos participantes em torno dos efeitos gerados em relação as suas práticas parentais, habilidades sociais, autorregulação das emoções, limites e gerenciamento dos comportamentos indesejáveis nos filhos" (GUISSO; BOLZE; VIERA, 2019. p. 247). Em um estudo de Casali (2019) sobre programas de habilidades sociais com pais e professores, o autor apresentou resultados que sugerem que os programas são efetivos, ao passo em que contribuem de forma indireta para o desenvolvimento socioemocional das crianças. Desta forma, são registradas diferenças significativas de antes e após a intervenção, no que se refere ao contexto de habilidades sociais educativas.

Santos e Wachelke (2019) efetuaram uma revisão bibliográfica narrativa sobre as habilidades sociais dos pais e os comportamentos dos filhos, concluindo-se que pais habilidosos "influenciam na competência social e acadêmica dos filhos, no comportamento adequado" (SANTOS; WACHELKE, 2019, p. 13). Assim como os programas de treinamento de habilidades sociais e habilidades parentais "mostram-se eficazes na melhora do repertório social de pais e filhos, bem como na relação entre eles" (SANTOS; WACHELKE, 2019, p. $1)$.

\section{Abrangência e limites da psicoeducação a pais ou cuidadores e sua relação com habilidades sociais}

Conforme, estudos descritos acima pode se considerar que, a psicoeducação tem sido uma técnica bastante utilizada e com apontamentos de eficácia em variáveis como cuidadores de pessoas com transtornos psicológicos, diferentes tipos de doenças e demência. Com eficácia também em grupos e em conjunto com mais técnicas cognitivas comportamentais. E intervenção direta com pessoas com transtornos psicológicos.

Pode se destacar alguns benefícios como autoconsciência e automotivação, assim como a empatia e gestão de emoções e saúde mental (COELHO, 2012); bem-estar, aquisição de estratégias de enfrentamento, senso de autoeficácia, diminuindo sentimentos e pensamentos disfuncionais (LOPES; CACHIONI, 2012); compreensão para aceitação e adesão ao tratamento e melhora na qualidade das atividades diárias necessárias para a saúde e qualidade de vida (MENEZES; SOUZA, 2012); autonomia, lazer e também relacionamentos interpessoais (NOGUEIRA; CRISOSTOMO, 2017); melhora na qualidade de vida, na comunicação, na colaboração, na forma de lidar com os problemas e atividades diárias e, diminuição da desesperança (SILVA; SÁ; SOUZA, 2018); diminui a frequência de recaída, o 
estigma em relação a saúde mental e aumenta o empoderamento da família e; mudanças positivas nas práticas dos pais (VALENTE et al., 2019).

Quanto a sua eficácia na utilização em intervenção em grupo, deve se considerar os atributos da intervenção em grupo. Logo, resultados positivos encontrados nos estudos nesse contexto, pode-se destacar a troca de experiências proporcionando redes de apoio assim como abre para mais formas de enxergar as situações e acrescentando aquisição de habilidades (CUEVAS-CANCINO; MORENO-PÉREZ, 2017). E quanto a sua utilização com demais técnicas cognitivas comportamentais os resultados dos estudos revelam melhoria na qualidade de vida, redução de ansiedade e depressão, maior percepção de apoio, dentre outros.

Em intervenção direta a pacientes com transtorno psicológicos se verificou que a psicoeducação pode impactar positivamente no bem-estar por promoção de hábitos saudáveis (PELLEGRINI, 2010). Com o uso da psicoeducação considera-se que à medida que passam a ter conhecimento passam a observar como funcionam podendo assim melhorar a motivação para a mudança (KNAPP et al., 2007). Contudo um estudo dentre os utilizados apontou maior eficácia em intervenção breve motivacional do que psicoeducação para as mudanças positivas nas práticas dos pais em monitoramento, abuso físico e punição não condizente (VALENTE et al., 2019). Algumas limitações dos estudos são apontadas pelos autores como falta de padronização de estrutura, de duração e de conteúdo. Assim como sugestões de avaliar a necessidade de manutenção ao longo do tempo em relação aos programas psicoeducativos e aos estudos aumento do número de seções e replicamento.

Vários estudos se debruçam sobre os estilos parentais e sua relação com o desenvolvimento ou influência sobre os comportamentos e habilidades das crianças. Conforme Mondin (2008) pais autoritativos em que predomina exigência e responsividade adequada, com comunicação clara e aberta e com respeito mútuo possibilita a desenvolver habilidades sociais nos filhos. Podemos destacar algumas correlações como comunicação e expressão que se associa de forma positiva a habilidades sociais e, estabelecer limites se associa de forma negativa a respostas externalizantes e problemas comportamentais (VILASBOAS; BOLSONI-SILVA, 2010). Indicativo positivo de relação entre práticas educativas parentais como expressão de sentimentos, enfrentamento assim como comunicação e poucas práticas negativas a filhos mais habilidosos e com menos problemas de comportamento (LEME; BOLSONI-SILVA, 2010). Práticas parentais com afeto e controle adequado tem uma relação positiva com competência acadêmica (MARTINS et al., 2014).

O estilo autoritário característico de autocontrole e baixa responsividade prezam respeito e obediência, utilizando com mais frequência punição, não valorizando diálogo, a 
autonomia e opiniões dos filhos (MONDIN, 2008). Encontra se correlações sobre o controle psicológico uma relação negativa com habilidades sociais (MARTINS et al., 2014). E práticas negativas relacionadas a problema de comportamento (BOLSONI-SILVA; LOUREIRO, 2019). O indulgente com baixo controle e alta responsividade, sem estabelecimento de regras e limites. O negligente com baixo controle e responsividade, não se envolvem com os filhos, nem exigem, nem são afetivos (MONDIN, 2008). Silva et al. (2005) constata relação entre problemas de comportamento e competência social ligados à negligência e monitoria inadequada (falta ou excesso). Técnicas coercivas utilizadas por alguns estilos parentais com mais frequência como autoritário e falta de afeto como também no negligente, podem gerar nas crianças medo, raiva e ansiedade e dificuldade de compreender a situação e o motivo da mudança no comportamento (MONDIN, 2008).

Estudos com programas de treinamentos de pais obtiveram resultados positivos nas habilidades sociais educativas parentais, assim como mudanças positivas nos filhos (COELHO; MURTA, 2007); no repertório social dos pais e filhos e na relação entre eles (SANTOS; WACHELKE, 2019); mudanças de comportamento parental e melhora nos comportamentos dos filhos, reduzindo práticas negativas e aumentando as positivas (GUISSO; BOLZE; VIERA, 2019). Assim como um programa desenvolvido a pais e professores sugere contribuição de forma indireta ao desenvolvimento socioemocional das crianças (CASALI, 2019).

Outro estudo verifica que habilidades dos professores como competência em conversar e estabelecer regras e limites podem melhorar habilidades sociais e problemas de comportamento em crianças (OLIVEIRA; LOUREIRO, 2019). E professores que apresentaram comportamentos habilidosos parecem promover habilidades nas crianças de forma indireta por imitação e emissão de feedback (MARIANO; BOLSONI-SILVA, 2016). São encontradas variáveis demográficas, conjugalidade e depressão materna correlacionado com comportamentos das crianças (BOLSONI-SILVA; LOUREIRO, 2019). Por exemplo, a separação tende a produzir menos problemas quando são estabelecidas interações entre pais e filhos satisfatórias (VILAS-BOAS; BOLSONI-SILVA, 2010). E a forma como ocorre a aprendizagem dos comportamentos desejáveis e indesejáveis pode ser por instrução, consequenciação e imitação podendo ser em sequência planejada ou aleatória (DEL PRETE; DEL PRETE, 2018).

\section{Considerações finais}


Pais ou cuidadores que atendem as necessidades físicas ou psicológicas das crianças ou adolescentes podem ser considerados pais ou cuidadores com práticas ou estilos sociais educativos parentais habilidosos. Neste caso, eles sabem se comunicar e se expressar, estabelecer limites, expressar sentimentos e executar controle adequado as situações. Pais ou cuidadores habilidosos tendem a desenvolver nas crianças, com as quais convivem, habilidades sociais, competência, assertividade, autonomia, facilidade em comunicação e expressão.

As necessidades básicas emocionais de Young como vínculos seguros, autonomia e competência, limites realistas, necessidades e suas emoções validadas e sentimentos expressos de forma assertiva e espontaneidade das escolhas, sendo supridas de forma adequada tendem a desenvolver modos mais assertivos. Assim como constatam estudos em relação entre Habilidades Sociais nas crianças, relacionado a estilos educativos parentais, habilidosos. Sendo que, conforme Knapp et al. (2007), à medida que passam a ter conhecimento passam a observar como funcionam e melhorar a motivação para a mudança.

Logo, quanto à utilização da psicoeducação pode-se considerar relevante. Se levar em consideração os estudos que consideram o seu uso eficaz a autoconsciência e automotivação, compreensão, aceitação, colaboração dentre outros. Estudos sobre intervenções a pais ou cuidadores ou professores constatam mudanças nas habilidades educativas parentais provocam mudanças nos comportamentos dos filhos. Assim como psicoeducação em intervenção direta ao paciente, e, seu uso com professores, pois as crianças e adolescentes podem sofrer influências na sua construção do repertório de habilidades através do convívio fora do contexto familiar, por exemplo, na escola.

\section{REFERÊNCIAS}

BOLSONI-SILVA, A. T.; SILVEIRA, F. F.; MARTURANO, E. M. Promovendo habilidades sociais educativas parentais na prevenção de problemas de comportamento. Rev. bras. ter. comport. cogn., Belo Horizonte, v. 10, n. 2, p. 125-142, 2008. Disponível em: http://pepsic.bvsalud.org/pdf/rbtcc/v10n2/v10n2a02.pdf. Acesso em: 31 jul. 2019.

BOLSONI-SILVA, A. T; LOUREIRO, S. R.; MARTURANO, E. M. Comportamentos internalizantes: associações com habilidades sociais, práticas educativas, recursos do ambiente familiar e depressão materna. Revista Psico., Porto Alegre, v. 47, n. 2, p. 111-120, 2016. DOI: http://dx.doi.org/10.15448/1980-8623.2016.2.20806

BOLSONI-SILVA, A. T.; LOUREIRO, S. R. Práticas Parentais: Conjugalidade, Depressão Materna, Comportamento das Crianças e Variáveis Demográficas. Revista Psico-USF, 
Campinas, v. 24, n. 1, p. 69-83, jan. 2019. DOI: http://dx.doi.org/10.1590/141382712019240106

BRANDÃO, T.; MATOS, P. M. Eficácia das intervenções psicológicas em grupo dirigidas a mulheres com cancro da mama: uma revisão sistemática. Rev. Port. Sau. Pub., v. 33, n. 1, p. 98-106, jan./jun. 2015. DOI: http://dx.doi.org/10.1016/j.rpsp.2015.01.001

CABALLO, V. E. Manual de avaliação e treinamento de habilidades sociais. São Carlos, SP: Livraria e Ed. Santos, 1. ed. 2003. p. 1-408.

CASALI, I. G. Programa de habilidades sociais com pais e professores: efeitos sobre educadores e crianças escolares. 2019. 249 f. Tese (Doutorado em Psicologia) - Centro de Educação de Ciências Humanas, Universidade Federal de São Carlos, São Carlos, 2019. Disponível em:

https://repositorio.ufscar.br/bitstream/handle/ufscar/11554/Tese\%20Ivana.pdf?sequence=1. Acesso em: 31 jul. 2019.

COELHO, L. V. M. Competência Emocional em Professores: Contributos da Psicoeducação. Revista Portuguesa de Enfermagem de Saúde Mental, Porto (Portugal), n. 8, p. 16-24, dez. 2012. Disponível em: http://www.scielo.mec.pt/scielo.php?script=sci_arttext\&pid=S1647$21602012000200003 \& \operatorname{lng}=$ pt\&nrm=iso. Acesso em: 31 jul. 2019.

COELHO, M. V.; MURTA, S. G. Treinamento de pais em grupo: um relato de experiência. Revista Estud. Psicol., Campinas, v. 24, n. 3, p. 333-341, jul./set. 2007. DOI: http://dx.doi.org/10.1590/S0103-166X2007000300005

CUEVAS-CANCINO, J. J.; MORENO-PÉREZ, N. E. Psicoeducação: intervenção de enfermagem para o cuidado da família em seu rolo de cuidadora. Enfermería Universitaria., México, v. 14, n. 3, p. 207-218, jul. 2017. Disponível em: http://www.scielo.org.mx/pdf/eu/v14n3/2395-8421-eu-14-03-00207.pdf. Acesso em: 31 jul. 2019.

DEL PRETE, A.; DEL PRETE, Z. A. P. (Org). Habilidades sociais, desenvolvimento e aprendizagem: questões conceituais, avaliação e intervenção. 1. ed. Campinas: Alínea, 2003.

DEL PRETE, A.; DEL PRETE, Z. A. P. A relação entre habilidades sociais e análise do comportamento: história e atualidades. In: KIENEN, K.; GIL, S. R. S. A.; LUZIA, J. C.; GAMBA, J. (Org.). Análise do comportamento: conceitos e aplicações a processos educativos clínicos e organizacionais. Londrina: UEL, 2018. p. 39-53. Disponível em: https://www.researchgate.net/publication/330666591_A_relacao_entre_habilidades_sociais_e _analise_do_comportamento_historia_e_atualidades. Acesso em: 31 jul. 2019.

GUISSO, L.; BOLZE, S. D. A.; VIERA, M. L. Práticas parentais positivas e programas de treinamento parental: uma revisão sistemática da literatura. Contextos Clínicos, v. 12, n. 1, jan./abr. 2019. DOI: http://dx.doi.org/10.4013/ctc.2019.121.10

KNAPP, P. et al. Terapia cognitivo-comportamental na prática psiquiátrica. Porto Alegre: Artmed, 2007. 520 p. 
LEME, V. B. R. \& BOLSONI-SILVA, A. T. Habilidades sociais educativas parentais e comportamento de pré-escolares. Estudos de psicologia, Natal, v. 15, n. 2, 161-173, maio/ago. 2010. DOI: https://doi.org/10.1590/S1413-294X2010000200005

LOPES, L. O.; CACHIONI, M. Intervenções psicoeducacionais para cuidadores de idosos com demência: uma revisão sistemática. J. bras. psiquiatr., Rio de Janeiro, v. 61, n. 4, p. 252-261, 2012. DOI: http://dx.doi.org/10.1590/S0047-20852012000400009

MARIANO, M.; BOLSONI-SILVA, A. T. Comparações entre práticas educativas de professores, habilidades sociais e problemas de comportamento de alunos. Revista Estudos e Pesquisas em Psicologia, Rio de Jneiro, v. 16, n. 1, p. 140-160, 2016. Disponível em: http://www.redalyc.org/articulo.oa?id=451846425009. Acesso em: 31 jul. 2019.

MARTINS, R. P et al. Práticas parentais: associações com desempenho escolar e habilidades sociais. Revista Psicol. Argum., Curitiba, v. 32, n. 78, p. 89-100, jul./set. 2014. DOI: http://dx.doi.org/10.7213/psicol.argum.32.078.AO04

MENEZES, S. L.; SOUZA, M. C. B. M. Implicações de um grupo de Psicoeducação no cotidiano de portadores de Transtorno Afetivo Bipolar. Rev. esc. enferm. USP, São Paulo, v. 46, n. 1, p. 124-131, fev. 2012. DOI: http://dx.doi.org/10.1590/S0080-62342012000100017

NOGUEIRA, C. A.; CRISOSTOMO, K. N.; SOUZA, R. S.; PRADO, J. M. A importância da psicoeducação na terapia cognitivo- comportamental: Uma revisão sistemática. Revista das Ciências da Saúde do Oeste baiano - Hígia, Bahia, v. 2, n. 1, p. 108-120, 2017. Disponível em: http://www.fasb.edu.br/revista/index.php/higia/article/view/190/211. Acesso em: 31 jul. 2019.

MONDIN, E. M. C. Práticas educativas parentais e seus efeitos na criação dos filhos. Revista Psicol. Argum, v. 26, n. 54, p. 233-244, jul./set. 2008. Disponível em: https://periodicos.pucpr.br/index.php/psicologiaargumento/article/view/19885/19187. Acesso em: 31 jul. 2019.

OLIVEIRA, J. E. B.; LOUREIRO, S. R. Validação do Roteiro de Entrevista de Práticas Educativas para Professores (RE-HSE-Pr). Aval. psicol., Campinas, v. 18, n. 1, p. 76-85, jan./mar. 2019. DOI: http://dx.doi.org/10.15689/ap.2019.1801.14685.09

PELLEGRINI, K. B. Impacto da psicoeducação na recuperação sintomática e funcional dos pacientes bipolares. 2010. 168 f. Dissertação (Mestrado em Ciências) - Faculdade de Medicina, Universidade de São Paulo, São Paulo, 2010. Disponível em: https://www.teses.usp.br/teses/disponiveis/5/5142/tde-15042010112622/publico/KarinadeBarrosPellegrinelliMestrado.pdf. Acesso em: 31 jul. 2019.

PORTELLA, M. Estratégias de treinamento em habilidades sociais. Centro de Psicologia Aplicada e Formação/ Terapia Cognitiva comportamental. 1. ed. Rio de Janeiro: Santos, 2011. $408 \mathrm{p}$.

SALVO, C. G.; SILVARES, E. F. M.; TONI, P. M. Práticas educativas como forma de predição de problemas de comportamento e competência social. Revista Estud. Psicol., Campinas, v. 22, n. 2, p. 187-195, abr./jun. 2005. Disponível em: 
http://www.scielo.br/scielo.php?script=sci arttext\&pid=S0103-

166X2005000200008\&lng=en\&nrm=iso. Acesso em: 31 jul. 2019.

SANTOS, E. B.; WACHELKE, J. Relações entre habilidades sociais de pais e comportamento dos filhos: uma revisão da literatura. Pesquisas e Práticas Psicossociais, São João del-Rei, v. 14, n. 1, jan./mar. 2019. Disponível em:

http://pepsic.bvsalud.org/pdf/ppp/v14n1/12.pdf. Acesso em: 31 jul. 2019.

SILVA, M.; SÁ, L.; SOUZA L. Eficácia dos programas psicoeducacionais na sobrecarga dos familiares cuidadores de pessoas com demência: revisão integrativa. Revista Portuguesa de Enfermagem de Saúde Mental, Porto (Portugal), v. 55, n. 19 p. 54-60, jun. 2018. DOI: http://dx.doi.org/10.19131/rpesm.0202

VALENTE, J. Y. et al. Ensaio clínico rondomizado para mudar as práticas de uso de drogas dos pais em um grupo de tele saúde de prevenção: estudo piloto. (Versão em português). J Pediatr, Rio de Janeiro, v. 95, n. 3, p. 334-341, maio/jun. 2019. DOI: https://doi.org/10.1016/j.jped.2018.02.004

VILAS-BOAS, A. C. V. B.; BOLSONI-SILVA, A. T. Habilidades sociais educativas de mães separadas e sua relação com o comportamento de pré-escolares. Revista Psico-USF (Impr), Campinas, v. 15, n. 3, p. 301-310, set./dez. 2010. DOI: http://dx.doi.org/10.1590/S141382712010000300004

YOUNG, J. E. Terapia do esquema: guia de técnicas cognitivo comportamentais inovadoras. Trad: Costa, R. C Porto Alegre: Artmed, 2008.

\section{Como referenciar este artigo}

CARDOSO, T. J. W.; QUEVEDO, R. F. Psicoeducação das necessidades básicas emocionais aos pais/ cuidadores e relação com habilidades sociais. Temas em Educ. e Saúde, Araraquara, v. 17, n. 00, p. e021004, 2021. e-ISSN 2526-3471. DOI: https://doi.org/10.26673/tes.v17i00.14410

Submetido em: 30/10/2020

Revisões requeridas: $10 / 12 / 2020$

Aceito em: 23/03/2021

Publicado em: 20/04/2021 